Georgian Mathematical Journal

Volume 12 (2005), Number 1, 131-138

\title{
UNIQUENESS OF NON-LINEAR DIFFERENTIAL POLYNOMIALS SHARING 1-POINTS
}

\author{
INDRAJIT LAHIRI AND PULAK SAHOO
}

\begin{abstract}
We prove two theorems on the uniqueness of nonlinear differential polynomials sharing 1-points which improve an earlier result of Fang and Hong.
\end{abstract}

2000 Mathematics Subject Classification: 30D35.

Key words and phrases: Uniqueness, meromorphic function, non-linear differential polynomial.

\section{Introduction, Definitions and Results}

Let $f$ and $g$ be two nonconstant meromorphic functions defined in the open complex plane $\mathbb{C}$. Let $k$ be a positive integer or infinity and $a \in\{\infty\} \cup \mathbb{C}$. We denote by $E_{k)}(a ; f)$ the set of all $a$-points of $f$ with multiplicities not exceeding $k$, where an $a$-point is counted according to its multiplicity. If for some $a \in$ $\{\infty\} \cup \mathbb{C}, E_{\infty)}(a ; f)=E_{\infty)}(a ; g)$, then we say that $f, g$ share the value $a \mathrm{CM}$ (counting multiplicities).

In $[3,5]$, the problem of uniqueness of meromorphic functions when two linear differential polynomials share the same 1-points was studied. Also in [3] the following question was asked: What can be said if two non-linear differential polynomials generated by two meromorphic functions share $1 C M$ ?

In the meantime some works have been done in this direction (cf. $[1,7]$ ). Recently, Fang and Hong [1] have proved the following result.

Theorem A ([1]). Let $f$ and $g$ be two transcendental entire functions and $n(\geq 11)$ be an integer. If $f^{n}(f-1) f^{\prime}$ and $g^{n}(g-1) g^{\prime}$ share $1 C M$, then $f \equiv g$.

In this paper we prove the following two theorems which improve Theorem A.

Theorem 1.1. Let $f$ and $g$ be two transcendental entire functions and $n(\geq 7)$ be an integer. If $E_{3)}\left(1 ; f^{n}(f-1) f^{\prime}\right)=E_{3)}\left(1 ; g^{n}(g-1) g^{\prime}\right)$, then $f \equiv g$.

Theorem 1.2. Let $f$ and $g$ be two transcendental meromrophic functions such that $\Theta(\infty ; f)>0, \Theta(\infty ; g)>0, \Theta(\infty ; f)+\Theta(\infty ; g)>\frac{4}{n+1}$, and $n(\geq 11)$ be an integer. If $E_{3)}\left(1 ; f^{n}(f-1) f^{\prime}\right)=E_{3)}\left(1 ; g^{n}(g-1) g^{\prime}\right)$, then $f \equiv g$.

Remark 1.1. If we choose $n(\geq 12)$, then in Theorem 1.2 the condition $\Theta(\infty ; f)>0$ and $\Theta(\infty ; g)>0$ can be removed.

The following example shows that the condition $\Theta(\infty ; f)+\Theta(\infty ; g)>\frac{4}{n+1}$ is sharp for Theorem 1.2. 
Example 1.1. Let

$$
f=\frac{(n+2)\left(1-h^{n+1}\right)}{(n+1)\left(1-h^{n+2}\right)}, \quad g=\frac{(n+2) h\left(1-h^{n+1}\right)}{(n+1)\left(1-h^{n+2}\right)} \quad \text { and } \quad h=\frac{\alpha^{2}\left(e^{z}-1\right)}{e^{z}-\alpha},
$$

where $\alpha=\exp \left(\frac{2 \pi i}{n+2}\right)$ and $n$ is a positive integer.

Then $T(r, f)=(n+1) T(r, h)+O(1)$ and $T(r, g)=(n+1) T(r, h)+O(1)$. Further we see that $h \neq \alpha, \alpha^{2}$ and a root of $h=1$ is not a pole of $f$ and $g$. Hence $\Theta(\infty ; f)=\Theta(\infty ; g)=2 /(n+1)$. Also, $f^{n+1}\left(\frac{f}{n+1}-\frac{1}{n+1}\right) \equiv g^{n+1}\left(\frac{g}{n+1}-\frac{1}{n+1}\right)$ and $f^{n}(f-1) f^{\prime} \equiv g^{n}(g-1) g^{\prime}$ but $f \not \equiv g$.

Though we do not explain the standard notation of the value distribution theory (see [2]) we give the following definitions.

Definition $1.1([4])$. For $a \in \mathbb{C} \cup\{\infty\}$ we denote by $N(r, a ; f \mid=1)$ the counting functions of simple $a$-points of $f$.

For a positive integer $m$ we denote by $N(r, a ; f \mid \leq m)(N(r, a ; f \mid \geq m))$ the counting function of those $a$-points of $f$ whose multiplicities are not greater(less) than $m$, where each $a$-point is counted according to its multiplicity.

$\bar{N}(r, a ; f \mid \leq m)$ and $\bar{N}(r, a ; f \mid \geq m)$ are defined similarly, where in counting the $a$-points of $f$ we ignore the multiplicities.

Also $N(r, a ; f \mid<m), N(r, a ; f \mid>m), \bar{N}(r, a ; f \mid<m)$ and $\bar{N}(r, a ; f \mid>m)$ are defined analogously.

Definition 1.2 (cf. [12]). For $a \in \mathbb{C} \cup\{\infty\}$ we put

$$
N_{2}(r, a ; f)=\bar{N}(r, a ; f)+\bar{N}(r, a ; f \mid \geq 2) .
$$

\section{LEMMAS}

In this section we present some lemmas which are needed in the sequel. We denote by $h$ the function

$$
h=\left(\frac{f^{\prime \prime}}{f^{\prime}}-\frac{2 f^{\prime}}{f-1}\right)-\left(\frac{g^{\prime \prime}}{g^{\prime}}-\frac{2 g^{\prime}}{g-1}\right) .
$$

Lemma 2.1. If $E_{1)}(1 ; f)=E_{1)}(1 ; g)$ and $h \not \equiv 0$, then

$$
N(r, 1 ; f \mid \leq 1) \leq N(r, 0 ; h) \leq N(r, h)+S(r, f)+S(r, g) .
$$

Proof. By a simple calculation we see that a simple zero of $f$ is a zero of $h$ and the lemma follows.

Lemma 2.2. If $E_{3)}(1 ; f)=E_{3)}(1 ; g)$ and $h \not \equiv 0$, then

$$
\begin{aligned}
N(r, h) \leq & \bar{N}(r, \infty ; f \mid \geq 2)+\bar{N}(r, 0 ; f \mid \geq 2)+\bar{N}(r, \infty ; g \mid \geq 2) \\
& +\bar{N}(r, 0 ; g \mid \geq 2)+\bar{N}(r, 1 ; f \mid \geq 4)+\bar{N}(r, 1 ; g \mid \geq 4) \\
& +\bar{N}_{0}\left(r, 0 ; f^{\prime}\right)+\bar{N}_{0}\left(r, 0 ; g^{\prime}\right),
\end{aligned}
$$

where $\bar{N}_{0}\left(r, 0 ; f^{\prime}\right)$ and $\bar{N}_{0}\left(r, 0 ; g^{\prime}\right)$ are the reduced counting functions of the zeros of $f^{\prime}$ and $g^{\prime}$ which are not the zeros of $f(f-1)$ and $g(g-1)$, respectively.

The proof is omitted. 
Lemma $2.3([13])$. If $h \equiv 0$, then $f$ and $g$ share $1 C M$.

Lemma 2.4 ([8, 10]). If $f$ and $g$ share $1 C M$, then one of the following cases holds:

(i) $T(r, f)+T(r, g) \leq 2\left\{N_{2}(r, 0 ; f)+N_{2}(r, 0 ; g)+N_{2}(r, \infty ; f)+N_{2}(r, \infty ; g)\right\}+$ $S(r, f)+S(r, g)$,

(ii) $f \equiv g$;

(iii) $f g \equiv 1$.

Lemma 2.5. If $E_{3)}(1 ; f)=E_{3)}(1 ; g)$, then the conclusion of Lemma 2.4 holds.

Proof. If $h \equiv 0$, then the result follows from Lemmas 2.3 and 2.4. So we suppose that $h \not \equiv 0$. Then by the second fundamental theorem, Lemmas 2.1 and 2.2 we get

$$
\begin{aligned}
T(r, f) \leq & \bar{N}(r, 0 ; f)+\bar{N}(r, 1 ; f)+\bar{N}(r, \infty ; f)-\bar{N}_{0}\left(r, 0 ; f^{\prime}\right)+S(r, f) \\
\leq & \bar{N}(r, 0 ; f)+\bar{N}(r, 1 ; f)+\bar{N}(r, \infty ; f)+\bar{N}(r, \infty ; f \mid \geq 2) \\
& +\bar{N}(r, 0 ; f \mid \geq 2)+\bar{N}(r, \infty ; g \mid \geq 2)+\bar{N}(r, 0 ; g \mid \geq 2) \\
& +\bar{N}(r, 1 ; f \mid \geq 4)+\bar{N}(r, 1 ; g \mid \geq 4)-N(r, 1 ; f \mid \leq 1) \\
& +\bar{N}_{0}\left(r, 0 ; g^{\prime}\right)
\end{aligned}
$$

Again, by the second fundamental theorem we get

$$
T(r, g) \leq \bar{N}(r, 0 ; g)+\bar{N}(r, \infty ; g)+\bar{N}(r, 1 ; g)-\bar{N}_{0}\left(r, 0 ; g^{\prime}\right)+S(r, g) \text {. }
$$

Also, we note that

$$
\bar{N}(r, 1 ; f)-\frac{1}{2} N(r, 1 ; f \mid \leq 1)+\bar{N}(r, 1 ; f \mid \geq 4) \leq \frac{1}{2} N(r, 1 ; f) \leq \frac{1}{2} T(r, f)
$$

and

$$
\bar{N}(r, 1 ; g)-\frac{1}{2} N(r, 1 ; g \mid \leq 1)+\bar{N}(r, 1 ; g \mid \geq 4) \leq \frac{1}{2} N(r, 1 ; g) \leq \frac{1}{2} T(r, g) .
$$

Adding (2.1) and (2.2) and using (2.3) and (2.4), we obtain

$$
\begin{aligned}
T(r, f)+T(r, g) \leq & 2\left\{N_{2}(r, 0 ; f)+N_{2}(r, \infty ; f)+N_{2}(r, 0 ; g)+N_{2}(r, \infty ; g)\right\} \\
& +S(r, f)+S(r, g) .
\end{aligned}
$$

This proves the lemma.

Lemma 2.6. Let $f$ and $g$ be two nonconstant meromorphic functions such that

$$
\Theta(\infty ; f)+\Theta(\infty ; g)>\frac{4}{n+1}
$$

where $n(\geq 2)$ is an integer. Then

$$
f^{n+1}(a f+b) \equiv g^{n+1}(a g+b)
$$

implies $f \equiv g$, where $a, b$ are finite nonzero constants and $n$ is an integer.

We omit the proof because it can be carried out that of Lemma 6 [6]. 
Lemma 2.7. Let $f$ and $g$ be nonconstant meromorphic functions. Then

$$
f^{n}(f-1) f^{\prime} g^{n}(g-1) g^{\prime} \not \equiv 1,
$$

where $n(\geq 5)$ is an integer.

Proof. If possible, let

$$
f^{n}(f-1) f^{\prime} g^{n}(g-1) g^{\prime} \equiv 1 .
$$

Let $z_{0}$ be an 1 -point of $f$ with multiplicity $p(\geq 1)$. Then $z_{0}$ is a pole of $g$ with multiplicity $q(\geq 1)$ such that

$$
2 p-1=(n+2) q+1
$$

i.e.,

$$
2 p=(n+2) q+2 \geq n+4
$$

i.e.,

$$
p \geq \frac{n+4}{2} .
$$

Let $z_{0}$ be a zero of $f$ with multiplicity $p(\geq 1)$ and it be a pole of $g$ with multiplicity $q(\geq 1)$. Then

$$
(n+1) p-1=(n+2) q+1 .
$$

From (2.5) we get

$$
\begin{aligned}
& q+2=(n+1)(p-q) \geq n+1 \\
\text { i.e., } & q \geq n-1 .
\end{aligned}
$$

Again, from(2.5) we get

$$
\begin{aligned}
&(n+1) p=(n+2) q+2 \geq(n+2)(n-1)+2 \\
& \text { i.e., } \quad p \geq \frac{(n+2)(n-1)+2}{n+1}=n .
\end{aligned}
$$

Since a pole of $f$ is either a zero of $g(g-1)$ or a zero of $g^{\prime}$, we see that

$$
\begin{aligned}
\bar{N}(r, \infty ; f) & \leq \bar{N}(r, 0 ; g)+\bar{N}(r, 1 ; g)+\bar{N}_{0}\left(r, 0 ; g^{\prime}\right) \\
& \leq \frac{1}{n} N(r, 0 ; g)+\frac{2}{n+4} N(r, 1 ; g)+\bar{N}_{0}\left(r, 0 ; g^{\prime}\right) \\
& \leq\left(\frac{1}{n}+\frac{2}{n+4}\right) T(r, g)+\bar{N}_{0}\left(r, 0 ; g^{\prime}\right) .
\end{aligned}
$$

Now by the second fundamental theorem we obtain

$$
\begin{aligned}
T(r, f) & \leq \bar{N}(r, 0 ; f)+\bar{N}(r, 1 ; f)+\bar{N}(r, \infty ; f)-\bar{N}_{0}\left(r, 0 ; f^{\prime}\right)+S(r, f) \\
& \leq \frac{1}{n} N(r, 0 ; f)+\frac{2}{n+4} N(r, 1 ; f)+\bar{N}(r, \infty ; f)-\bar{N}_{0}\left(r, 0 ; f^{\prime}\right)+S(r, f),
\end{aligned}
$$


i.e.,

$$
\begin{aligned}
\left(1-\frac{1}{n}-\frac{2}{n+4}\right) T(r, f) \leq & \left(\frac{1}{n}+\frac{2}{n+4}\right) T(r, g)+\bar{N}_{0}\left(r, 0 ; g^{\prime}\right) \\
& -\bar{N}_{0}\left(r, 0 ; f^{\prime}\right)+S(r, f) .
\end{aligned}
$$

Similarly, we get

$$
\begin{aligned}
\left(1-\frac{1}{n}-\frac{2}{n+4}\right) T(r, g) \leq & \left(\frac{1}{n}+\frac{2}{n+4}\right) T(r, f)+\bar{N}_{0}\left(r, 0 ; f^{\prime}\right) \\
& -\bar{N}_{0}\left(r, 0 ; g^{\prime}\right)+S(r, g) .
\end{aligned}
$$

Adding (2.6) and (2.7) we get

$$
\left(1-\frac{2}{n}-\frac{4}{n+4}\right)\{T(r, f)+T(r, g)\} \leq S(r, f)+S(r, g),
$$

which is a contradiction because $1-\frac{2}{n}-\frac{4}{n+4}>0$. This proves the lemma.

Lemma $2.8([9])$. Let $f$ be a nonconstant meromorphic function and $P(f)=$ $a_{0}+a_{1} f+a_{2} f^{2}+\cdots+a_{n} f^{n}$, where $a_{0}, a_{1}, \ldots, a_{n}$ are constants and $a_{n} \neq 0$. Then

$$
T(r, P(f))=n T(r, f)+O(1) .
$$

Lemma 2.9 ([11]). Let $f$ be a nonconstant meromorphic function. Then

$$
N\left(r, 0 ; f^{(k)}\right) \leq k \bar{N}(r, \infty ; f)+N(r, 0 ; f)+S(r, f) .
$$

Lemma 2.10. Let $f$ and $g$ be two nonconstant meromorphic functions and

$$
F=f^{n+1}\left(\frac{f}{n+2}-\frac{1}{n+1}\right) \text { and } G=g^{n+1}\left(\frac{g}{n+2}-\frac{1}{n+1}\right)
$$

where $n(\geq 4)$ is an integer. Then $F^{\prime} \equiv G^{\prime}$ implies $F \equiv G$.

Proof. If $F^{\prime} \equiv G^{\prime}$ then $F \equiv G+c$, where $c$ is a constant. If possible, let $c \neq 0$. Then by the second fundamental theorem and Lemma 2.8 we get

$$
\begin{aligned}
(n+2) T(r, f) \leq & \bar{N}(r, \infty ; F)+\bar{N}(r, 0 ; F)+\bar{N}(r, c ; F)+S(r, F) \\
= & \bar{N}(r, \infty ; f)+\bar{N}(r, 0 ; f)+\bar{N}\left(r, \frac{n+2}{n+1} ; f\right)+\bar{N}(r, 0 ; g) \\
& +\bar{N}\left(r, \frac{n+2}{n+1} ; g\right)+S(r, f) \\
\leq & 3 T(r, f)+2 T(r, g)+S(r, f),
\end{aligned}
$$

i.e.,

$$
(n-1) T(r, f) \leq 2 T(r, g)+S(r, f) .
$$

Similarly, we get

$$
(n-1) T(r, g) \leq 2 T(r, f)+S(r, g) \text {. }
$$

This shows that

$$
(n-3) T(r, f)+(n-3) T(r, g) \leq S(r, f)+S(r, g),
$$


which is a contradiction. Therefore $c=0$ and so $F \equiv G$. This proves the lemma.

\section{Proofs of the Theorems}

Proof of Theorem 1.2. Let $F$ and $G$ be defined as in Lemma 2.10. Now in view of the first fundamental theorem and Lemma 2.8 we get

$$
\begin{aligned}
T(r, F)= & T\left(r, \frac{1}{F}\right)+O(1) \\
= & N(r, 0 ; F)+\left(r, \frac{1}{F}\right)+O(1) \\
\leq & N(r, 0 ; F)+m\left(r, \frac{F^{\prime}}{F}\right)+m\left(r, \frac{1}{F^{\prime}}\right) \\
= & T\left(r, F^{\prime}\right)+N(r, 0 ; F)-N\left(r, 0 ; F^{\prime}\right)+S(r, F) \\
= & T\left(r, F^{\prime}\right)+(n+1) N(r, 0 ; f)+N\left(r, \frac{n+2}{n+1} ; f\right)-n N(r, 0 ; f) \\
& -N(r, 1 ; f)-N\left(r, 0 ; f^{\prime}\right)+S(r, f) \\
= & T\left(r, F^{\prime}\right)+N(r, 0 ; f)+N\left(r, \frac{n+2}{n+1} ; f\right) \\
& -N(r, 1 ; f)-N\left(r, 0 ; f^{\prime}\right)+S(r, f) .
\end{aligned}
$$

If possible, suppose that

$$
\begin{aligned}
T\left(r, F^{\prime}\right)+T\left(r, G^{\prime}\right) \leq & 2\left\{N_{2}\left(r, 0 ; F^{\prime}\right)+N_{2}\left(r, 0 ; G^{\prime}\right)+N_{2}\left(r, \infty ; F^{\prime}\right)\right. \\
& \left.+N_{2}\left(r, \infty ; G^{\prime}\right)\right\}+S\left(r, F^{\prime}\right)+S\left(r, G^{\prime}\right) .
\end{aligned}
$$

Then we get by Lemma 2.9

$$
\begin{aligned}
T(r, F)+ & T(r, G) \leq T\left(r, F^{\prime}\right)+T\left(r, G^{\prime}\right)+N(r, 0 ; f)+N\left(r, \frac{n+2}{n+1} ; f\right) \\
& -N(r, 1 ; f)-N\left(r, 0 ; f^{\prime}\right)+N(r, 0 ; g)+N\left(r, \frac{n+2}{n+1} ; g\right) \\
& -N(r, 1 ; g)-N\left(r, 0 ; g^{\prime}\right)+S(r, f)+S(r, g) \\
\leq & 2 N_{2}\left(r, 0 ; F^{\prime}\right)+2 N_{2}\left(r, 0 ; G^{\prime}\right)+2 N_{2}\left(r, \infty ; F^{\prime}\right) \\
& +2 N_{2}\left(r, \infty ; G^{\prime}\right)+N(r, 0 ; f)+N\left(r, \frac{n+2}{N+1} ; f\right) \\
& -N(r, 1 ; f)-N\left(r, 0 ; f^{\prime}\right)+N(r, 0, g)+N\left(r, \frac{n+2}{n+1} ; g\right) \\
& -N(r, 1 ; g)-N\left(r, 0 ; g^{\prime}\right)+S(r, f)+S(r, g) \\
\leq & 4 \bar{N}(r, 0 ; f)+2 N(r, 1 ; f)+2 N\left(r, 0 ; f^{\prime}\right) \\
& +4 \bar{N}(r, 0 ; g)+2 N(r, 1 ; g)+2 N\left(r, 0 ; g^{\prime}\right) \\
& +4 \bar{N}(r, \infty ; f)+4 \bar{N}(r, \infty ; g)+N(r, 0 ; f)
\end{aligned}
$$


UNIQUENESS OF NON-LINEAR DIFFERENTIAL POLYNOMIALS

$$
\begin{aligned}
& +N\left(r, \frac{n+2}{n+1} ; f\right)-N(r, 1 ; f)-N\left(r, 0 ; f^{\prime}\right) \\
& +N(r, 0 ; g)+N\left(r, \frac{n+2}{n+1} ; g\right)-N(r, 1 ; g)-N\left(r, 0 ; g^{\prime}\right) \\
& +S(r, f)+S(r, g) \\
& \leq 6 N(r, 0 ; f)+N(r, 1 ; f)+5 \bar{N}(r, \infty ; f)+N\left(r, \frac{n+2}{n+1} ; f\right) \\
& +6 N(r, 0 ; g)+N(r, 1 ; g)+5 \bar{N}(r, \infty ; g)+N\left(r, \frac{n+2}{n+1} ; g\right) \\
& +S(r, f)+S(r, g) \\
& \leq \\
& +5 T(r, f)+8 T(r, g)+5 \bar{N}(r, \infty ; f)+5 \bar{N}(r, \infty ; g) \\
& +S(r, f)+S(r, g) .
\end{aligned}
$$

So by Lemma 2.8 we obtain

$$
\begin{aligned}
(n-6) T(r, f) & +(n-6) T(r, g) \\
\leq & 5 \bar{N}(r, \infty ; f)+5 \bar{N}(r, \infty ; g)+S(r, f)+S(r, g) .
\end{aligned}
$$

Let us choose $\varepsilon$ such that

$$
0<\varepsilon<n-11+\min \{\Theta(\infty ; f), \Theta(\infty ; g)\}
$$

Then from (3.2) we get

$$
(n-11+\Theta(\infty ; f)-\varepsilon) T(r, f)+(n-11+\Theta(\infty ; g)-\varepsilon) T(r, g) \leq S(r, f)+S(r, g) \text {, }
$$

which is a contradiction.

Therefore inequality (3.1) does not hold. Since $E_{3)}\left(1 ; F^{\prime}\right)=E_{3)}\left(1 ; G^{\prime}\right)$, by Lemmas 2.5, 2.6, 2.7 and 2.10 we get $f \equiv g$. This proves the theorem.

Proof of Theorem 1.1. If (3.1) holds, then from (3.2) we get

$$
(n-6) T(r, f)+(n-6) T(r, g) \leq S(r, f)+S(r, g),
$$

which is a contradiction.

Therefore inequality (3.1) does not hold. Since $E_{3)}\left(1 ; F^{\prime}\right)=E_{3)}\left(1 ; G^{\prime}\right)$, by Lemmas 2.5, 2.6, 2.7 and 2.10 we get $f \equiv g$. This proves the theorem.

\section{REFERENCES}

1. M.-L. FAng and W. Hong, A unicity theorem for entire functions concerning differential polynomials. Indian J. Pure Appl. Math. 32(2001), No. 9, 1343-1348.

2. W. K. Hayman, Meromorphic functions. Oxford Mathematical Monographs Clarendon Press, Oxford, 1964.

3. I. LAHIRI, Uniqueness of meromorphic functions when two linear differential polynomials share the same 1-points. Ann. Polon. Math. 71(1999), No. 2, 113-128.

4. I. LAhiri, Value distribution of certain differential polynomials. Int. J. Math. Math. Sci. 28(2001), No. 2, 83-91. 
5. I. LAHIRI, Linear differential polynomials sharing the same 1-points with weight two. Ann. Polon. Math. 79(2002), No. 2, 157-170.

6. I. Lahiri, On a question of Hong Xun Yi. Arch. Math. (Brno) 38(2002), No. 2, 119-128.

7. W. C. Lin, Uniqueness of differential polynomials and a problem of Lahiri. (Chinese) Pure Appl. Math. (Xi'an) 17(2001), No. 2, 104-110.

8. E. Mues and M. Reinders, Meromorphic functions sharing one value and unique range sets. Kodai Math. J. 18(1995), No. 3, 515-522.

9. C. C. YANG, On deficiencies of differential polynomials. II. Math. Z. 125(1972), 107-112.

10. C. C. YAng and X. H. HuA, Uniqueness and value-sharing of meromorphic functions. Ann. Acad. Sci. Fenn. Math. 22(1997), No. 2, 395-406.

11. H. X. YI, Uniqueness of meromorphic functions and a question of C. C. Yang. Complex Variables Theory Appl. 14(1990), No. 1-4, 169-176.

12. H. X. YI, On characteristic function of a meromorphic function and its derivative. Indian J. Math. 33(1991), No. 2, 119-133.

13. H. X. YI, Some further results on uniqueness of meromorphic functions. Complex Variables Theory Appl. 38(1999), No. 4, 375-385.

(Received 9.03.2003)

Authors' address:

Department of Mathematics

University of Kalyani

West Bengal 741235

India

E-mails: ilahiri@hotmail.com

ilahiri@vsnl.com 